\title{
PREDATION, EFFICIENCY, AND INEQUALITY
}

\author{
Herschel I. Grossman \\ Minseong Kim
}

Working Paper 6301 


\title{
PREDATION, EFFICIENCY, AND INEQUALITY
}

\author{
Herschel I. Grossman \\ Minseong Kim
}

Working Paper 6301

http://www.nber.org/papers/w6301

\section{NATIONAL BUREAU OF ECONOMIC RESEARCH 1050 Massachusetts Avenue \\ Cambridge, MA 02138 \\ December 1997}

Any opinions expressed are those of the authors and not those of the National Bureau of Economic Research.

(C) 1997 by Herschel I. Grossman and Minseong Kim. All rights reserved. Short sections of text, not to exceed two paragraphs, may be quoted without explicit permission provided that full credit, including $\mathbb{C}$ notice, is given to the source. 
Predation, Efficiency, and Inequality

Herschel I. Grossman and Minseong Kim

NBER Working Paper No. 6301

December 1997

JEL Nos. D60, D74, D31, D50

\section{ABSTRACT}

This paper shows how predation breaks the links between an economy's aggregate resource endowment and aggregate consumption and between the interpersonal distribution of endowments and the interpersonal distribution of consumption. We construct a general-equilibrium model in which some people (the privileged) are well endowed with resources and other people (the unprivileged) are poorly endowed with resources and in which each person can choose to be either a producer or a predator. In this model the choice by some people to be predators decreases aggregate consumption, both because the resources of predators are wasted by not being used productively and because producers sacrifice production by allocating resources to guarding against predators.

Analyzing this model we find that the minimum equilibrium ratio of predators to producers depends only on the technology of predation. In addition, the equilibrium ratio of predators to producers equals its minimum value if and only if the ratio of unprivileged people to privileged people is not larger than this minimum value. These properties imply that, in contrast to a model that abstracts from predation, the fully egalitarian distribution of resources does not satisfy the Rawlsian criterion of maximizing the consumption of the person with the lowest consumption. (In fact, the fully egalitarian distribution is not even Pareto efficient). Instead, the Rawlsian criterion selects an unegalitarian distribution of resources in which the ratio of unprivileged people to privileged people equals the minimum ratio of predators to producers and in which unprivileged people have only the minimum possible endowment of resources. In the resulting Rawlsian equilibrium, only unprivileged people choose to be predators rather than producers, and because both the ratio of predators to producers and the amount of resources that predators waste are minimized, aggregate consumption is maximized. In addition, in the Rawlsian equilibrium predation equalizes the consumption of the privileged and the unprivileged.

Herschel I. Grossman

Department of Economics

Brown University

Box B

Providence, RI 02912

and NBER

herschel@brownvm.brown.edu
Minseong Kim

Department of Economics

University of Pittsburgh

Pittsburgh, PA 15260 
This paper shows how predation breaks the links between an economy's aggregate resource endowment and aggregate consumption and between the interpersonal distribution of endowments and the interpersonal distribution of consumption. We construct a generalequilibrium model in which some people (the privileged) are well endowed with resources and other people (the unprivileged) are poorly endowed with resources and in which each person can choose to be either a producer or a predator. Predators are people who produce nothing, but live by appropriating the product of the producers. ${ }^{1}$

We assume that each person chooses to be either a predator or a producer depending on whether predation or production yields more consumption for him. We also assume that the technologies of production and predation are such that the consumption that a person can obtain from being a producer is an increasing function of his resources, whereas the consumption that a person can obtain from being a predator does not depend on his resources. $^{2}$ In addition, if a person chooses to be a producer, then he must decide how to allocate his resources between production and guarding against predators. ${ }^{3}$ In making these

\footnotetext{
${ }^{1}$ This abstract analysis assumes that all activities can be classified either as being predatory, or as being directly productive, or as being a way to guard against predation. In fact, although some activities, such as burglary and robbery, are unambiguously predatory, and some activities, such as college teaching, are unambiguously productive, and some activities, such as installing locks, are unambiguously ways to guard against predation, there are activities, such as litigating, that are not easily classified. We leave the reader free to classify specific activities according to his or her own inclination. But, we point out that predation is not synonymous with crime. Although many predatory activities, like burglary and robbery, are criminal, many criminal activities, like illegal gambling and drug dealing, are productive and not predatory.X

${ }^{2}$ If human capital is an important component of a person's resources, then this assumption implies that our analysis is applicable to low-skilled predatory activities like burglary, robbery, and kidnapping and abstracts from high-skilled predatory activities like embezzlement and some forms of litigation.

${ }^{3}$ Dan Usher (1987) developed a pioneering general-equilibrium model in which people decide simultaneously whether to be producers or predators and in which producers also decide how much resources to allocate to guarding against predators. In Grossman and Kim (1997) we analyse the choices of people to be producers or predators in a model in which only some people, whom we define to be amoral, are potential
} 
choices each person takes the choices of all other people as given.

In our model the interpersonal distribution of resources has two dimensions. One dimension is the number of people who are unprivileged. The other dimension is the endowments of privileged and unprivileged people relative to the average endowment of resources. If either the number of unprivileged people approaches zero, or if the endowments of privileged and unprivileged people approach the average endowment, then we define the distribution of resources to be fully egalitarian.

We begin by analysing how the equilibrium configuration of choices depends on the technology of predation and on the interpersonal distribution of resources. We emphasize two properties of the equilibrium that are critical for the welfare analysis that follows: First, regardless of the interpersonal distribution of resources, the equilibrium ratio of predators to producers has a positive minimum value that depends only on the technology of predation. This property means that, even though a privileged person can produce more than an unprivileged person, and, as a result, predation is less attractive for the privileged than for the unprivileged, if the ratio of unprivileged people to privileged people were small, then not only all of the unprivileged people but also some of the privileged people would choose to be predators. Second, the equilibrium ratio of predators to producers exceeds its minimum value if and only if the ratio of unprivileged people to privileged people is larger than this minimum value.

Next, we show how the possibility of predation radically alters the welfare properties of the interpersonal distribution of resources. We apply the Rawlsian criterion, from John Rawls (1971), and we derive the interpersonal distribution of resources that maximizes the consumption of the person with the lowest consumption. This criterion is equivalent to maximizing the consumption of the each unprivileged person.

predators. The other people, whom we define to be moral, always choose to be producers, no matter how lucrative predation is relative to production. In the present paper we implicitly assume that everybody is amoral. Hence, every person is a potential predator. 
Our model is such that, if we were to ignore the possibility of predation, then aggregate consumption would depend only on the aggregate endowment of resources and the interpersonal distribution of consumption would correspond to the interpersonal distribution of resources. Accordingly, if we were to ignore the possibility of predation, then the Rawlsian criterion would select the fully egalitarian distribution of resources.

The fact that in equilibrium some people choose to be predators changes this conclusion for two reasons: First, the choice by some people to be predators decreases aggregate consumption, both because the resources of predators are wasted by not being used productively and because producers sacrifice production by allocating resources to guarding against predators. Second, predation increases the consumption of the unprivileged people relative to the consumption of the privileged people.

With some people choosing to be predators we find that the Rawlsian criterion selects an unegalitarian distribution of resources in which the ratio of unprivileged people to privileged people equals the minimum ratio of predators to producers, and the unprivileged people have only the minimum possible endowment of resources. In the resulting Rawlsian equilibrium, only unprivileged people choose to be predators, and, because both the ratio of predators to producers and the amount of resources that predators waste are minimized, aggregate consumption is maximized. In addition, in the Rawlsian equilibrium predation equalizes the consumption of the privileged and the unprivileged. We also show that, allowing for predation, the fully egalitarian distribution is not even Pareto efficient.

\section{Analytical Framework}

Assume that each unprivileged person has an endowment of $k$ units of resources, and that each privileged person has an endowment of $K$ units of resources, where $K \geq k \geq 0$. Also, assume that $u$, where $0 \leq u<1$, is the number of people who are unprivileged, and that $1-u$ is the number of people who are privileged, where we choose units such that the population is one. Let $U \equiv \frac{u}{1-u}$ denote the ratio of unprivileged people to privileged 
people. Aside from their endowments of resources, people are otherwise identical.

Given these numbers of privileged and unprivileged people, the average endowment of resources, denoted by $\Omega$, is

$$
\Omega=(1-u) K+u k \equiv \frac{K+U k}{1+U} .
$$

In analysing the welfare properties of the interpersonal distribution of resources, we hold $\Omega$ fixed. For a given viice of $\Omega$, the interpersonal distribution of resources can reflect any combination of $k, K$, and $u$ that satisfies equation (1).

Given his endowment of resources each person has to make two choices. First, he must choose whether to be a producer or a predator. Each person makes this choice individually, taking as given his potential consumption as a producer or as a predator. Let $N$ denote the number of people who are privileged and who choose to be producers, where $N \leq 1-u$, let $n$ denote the number of people who are unprivileged and who choose to be producers, where $n \leq u$, and let $r$ denote the number of people, whether privileged or unprivileged, who choose to be predators. Thus, we have $N+n+r=1$. Let $R \equiv \frac{r}{N+n}$ denote the ratio of predators to producers.

Second, if a person chooses to be a producer, then he must decide how to allocate his human capital between production and guarding against predators. Each producer also makes this choice individually, taking the ratio of predators to producers as given. Let $g$ denote the nonnegative fraction of his resources that a producer allocates to guarding against predators. ${ }^{4}$ Thus, $1-g$ denotes the nonnegative fraction of his resources that he allocates to production. Let $G \equiv \frac{g}{1-g}$ denote the ratio of the resources that a producer

\footnotetext{
${ }^{4}$ Guarding includes all actions that are costly but have the effect of decreasing the fraction of the production of consumables that predators appropriate. Examples of ways of guarding against predators include the locating of production in inconvenient but secure places, the production of things that are harder for predators to appropriate, the installation of locks, the building of walls, and the hiring of guards. For simplicity, the present analysis focuses on the total amount of resources allocated to guarding, abstracting from different ways of guarding.
} 
allocates to guarding against predators to the resources that he allocates to the production of consumables.

To simplify the analysis of the choice between being a producer and a predator, assume that a unit of resources can produce one unit of consumables. The number of units of consumables that a person actually produces equals the product of his endowment of resources and the fraction of his resources that he allocates to production. ${ }^{5}$

A producer appropriates the nonnegative fraction $p$ of the consumables that he produces, and predators appropriate the nonnegative fraction $1-p$. Assume that $p$ depends negatively on the ratio of predators to producers, $R$, and positively on $G$. Specifically,

$$
p=\frac{1}{1+\theta R / G}, \quad \theta \geq 0
$$

In equation (2), the parameter $\theta$, which embodies the technology of predation, determines the effectiveness of predators in appropriating consumables for given values of $R$ and $G$. The specification that $p$ depends on the number of predators but not on the identity of the predators reflects the assumption that privileged people and unprivileged people are equally effective at predation. ${ }^{6}$

Let $C$ denote the consumption of each privileged producer and let $c$ denote the consumption of each unprivileged producer. After allowing for the fraction of resources

${ }^{5}$ To focus on the effects of the possibility of predation, we assume that individual productive activities are independent, and we abstract from trade in either productive inputs or consumables.

${ }^{6}$ Equation (2) is a generic black box that conceals the process of predation, just as the standard generic production function conceals the process of production. For example, the relation between appropriative inputs and the appropriative outcome described by equation (2) could involve either the use of force or a peaceful settlement under the threat of force, although, strictly speaking, given complete information and the absence of stochastic factors, this model does not provide an internal explanation for costly violence. Dagobert Brito and Michael Intriligator (1985) address the question of whether appropriative conflict is resolved with or without violence and destruction, and emphasize the importance of incomplete information as a cause of violence. 
allocated to guarding against predators and for the fraction of consumables lost to predation, we have

$$
C=p(1-g) K \equiv \frac{p}{1+G} K \text { and } c=p(1-g) k \equiv \frac{p}{1+G} k
$$

Let $D$ denote the consumption of a predator. Assuming that each predator obtains an equal share of the total amount of consumables appropriated from the producers, we have ${ }^{7}$

$$
D=\frac{(1-p)(1-g)(N K+n k)}{r} .
$$

According to equation (4) $D$ is a decreasing function of $R$ and is well defined for positive values of $R$. Further, if $R$ equals zero, then the value of $D$ is defined to be $\lim _{R \rightarrow 0} D$, which, using equation (2), equals $\frac{\theta}{G}(1-g) K$.

\section{The Ratio of Predators to Producers}

To decide whether to be a producer or a predator, each privileged person compares the values of $C$ and $D$, taking $G$ and $R$ as given, and each unprivileged person compares the values of $c$ and $D$, taking $G$ and $R$ as given. The choices of privileged and unprivileged people to be producers or predators depend on $G$ in the following way:

1. $D>C>c$ : If $D$ were larger than both $c$ and $C$, then every person would prefer to be a predator. Substituting equation (2) into equations (3) and (4), we find that this case could occur if and only if $G$ were smaller than $\theta$. In this case, $R$ would be infinite.

2. $D=C>c$ : If $D$ equals $C$ but is larger than $c$, then unprivileged people prefer to be predators, whereas privileged people are indifferent between being producers or predators.

\footnotetext{
${ }^{7}$ For simplicity, the model assumes that predators only prey on producers. Predators do not prey on other predators. The model also abstracts from possible destruction of a some consumables as the result of predation. The models in Grossman and $\operatorname{Kim}(1995,1996)$ show how destruction is easily incorporated into the analysis. In addition, we could modify the model to allow the activity of guarding against predators to include the apprehension and punishment of predators. The apprehension and punishment of predators would not directly affect $p$, but would reduce the expected utility of predators.
} 
This case would occur if and only if $G$ equals $\theta$. In this case, $R$ can take any value larger than or equal to $U$.

3. $C>D>c$ : If $D$ is smaller than $C$ but larger than $c$, then unprivileged people prefer to be predators, whereas privileged people prefer to be producers. This case would occur only if $G$ is larger than $\theta$, but smaller than $\theta K / k$. In this case, $R$ is equal to $U$. 4. $C>D=c$ : If $D$ is smaller than $C$ but equal to $c$, then unprivileged people are indifferent between being producers or predators, whereas privileged people prefer to be producers. This case would occur only if $G$ is equal to or larger than $\theta \Omega / k$, but not larger than $\theta K / k$. In this case, the equality between $D$ and $c$ implies that $R$ is equal to $\frac{G-\theta \Omega / k}{\theta\left(\frac{\Omega}{k}-1\right)}$. This implied value of $R$ is equal to or smaller than $U$, but larger than or equal to zero.

5. $C>c>D$ : If $D$ were smaller than both $c$ and $C$, then every person would prefer to be a producer. This case could occur only if $G$ were larger than $\theta \Omega / k$. In this case, $R$ would be zero.

Summarizing these results, the choices of privileged and unprivileged people to be producers or predators are such that

$$
R=\left\{\begin{array}{lrl}
\infty & \text { if and only if } & G<\theta \\
x \in[U, \infty] & \text { if and only if } & G=\theta \\
U & \text { only if } & \theta<G<\theta K / k \\
\frac{G-\theta \Omega / k}{\theta\left(\frac{\Omega}{k}-1\right)} & \text { only if } & \theta \Omega / k \leq G \leq \theta K / k \\
0 & \text { only if } & G>\theta \Omega / k .
\end{array}\right.
$$

In Figures 1 and 2 the piecewise linear loci represent equation (5).

Each privileged person who chooses to be a producer chooses $G$ to maximize $C$, taking $R$ as given, and each unprivileged person who chooses to be a producer chooses $G$ to maximize $c$, taking $R$ as given. To analyse these choice problems we substitute equation (2) into equation (3) and calculate the value of the ratio $G$ that satisfies the conditions 
$d C / d G=0$ and $d c / d G=0$. For both privileged people and unprivileged people the solution to this problem is

$$
G=\sqrt{\theta R}
$$

In Figures 1 and 2 the smooth concave loci represent equation (6).

Solving equations (5) and (6) for $R$, and assuming that the ratio $k / \Omega$ is small, we find that in equilibrium ${ }^{8}$

$$
R=\max \{\theta, U\}
$$

Equation (7) reveals that, if $\theta$ is larger than $U$, then in equilibrium unprivileged people prefer to be predators, privileged people are indifferent between being producers and being predators, and $R$ equals $\theta$. Alternatively, if $U$ is larger than $\theta$, then in equilibrium unprivileged people prefer to be predators, privileged people prefer to be producers, and $R$ equals $U$. Figure 1 illustrates the case of $\theta>U$, and Figure 2 illustrates the case of $U>\theta$.

Two implications of equation (7) are essential for the welfare analysis that follows. The first implication is that, if $U$ is smaller than or equal to $\theta$, then in equilibrium $R$ equals $\theta$. The second implication is that, if $U$ is larger than $\theta$, then in equilibrium $R$ is larger

${ }^{8}$ More generally, solving equations (5) and (6) for $R$ would yield

$$
R= \begin{cases}\theta & \text { for } U \leq \theta \\ U & \text { for } \theta<U \leq R_{1} \\ R_{1} & \text { for } R_{1}<U<R_{2} \\ \left\{R_{1}, R_{2}, U\right\} & \text { for } U \geq R_{2},\end{cases}
$$

where $R_{1}$ and $R_{2}$ are the values of $R$ that satisfy both $R=\frac{G-\theta \Omega / k}{\theta\left(\frac{\Omega}{k}-1\right)}$, from equation (5), and $G=\sqrt{\theta R}$, from equation (6). Such values of $R$ would exist only if $k / \Omega$ were sufficiently large. Equation (7) assumes that $R_{1}$ and $R_{2}$ do not exist. Because both $R_{1}$ and $R_{2}$ would be larger than $\theta$, neither the two essential implications of equation (7) stressed below nor the conclusions drawn from the welfare analysis in the sections that follow depend on whether or not $R_{1}$ and $R_{2}$ exist. 


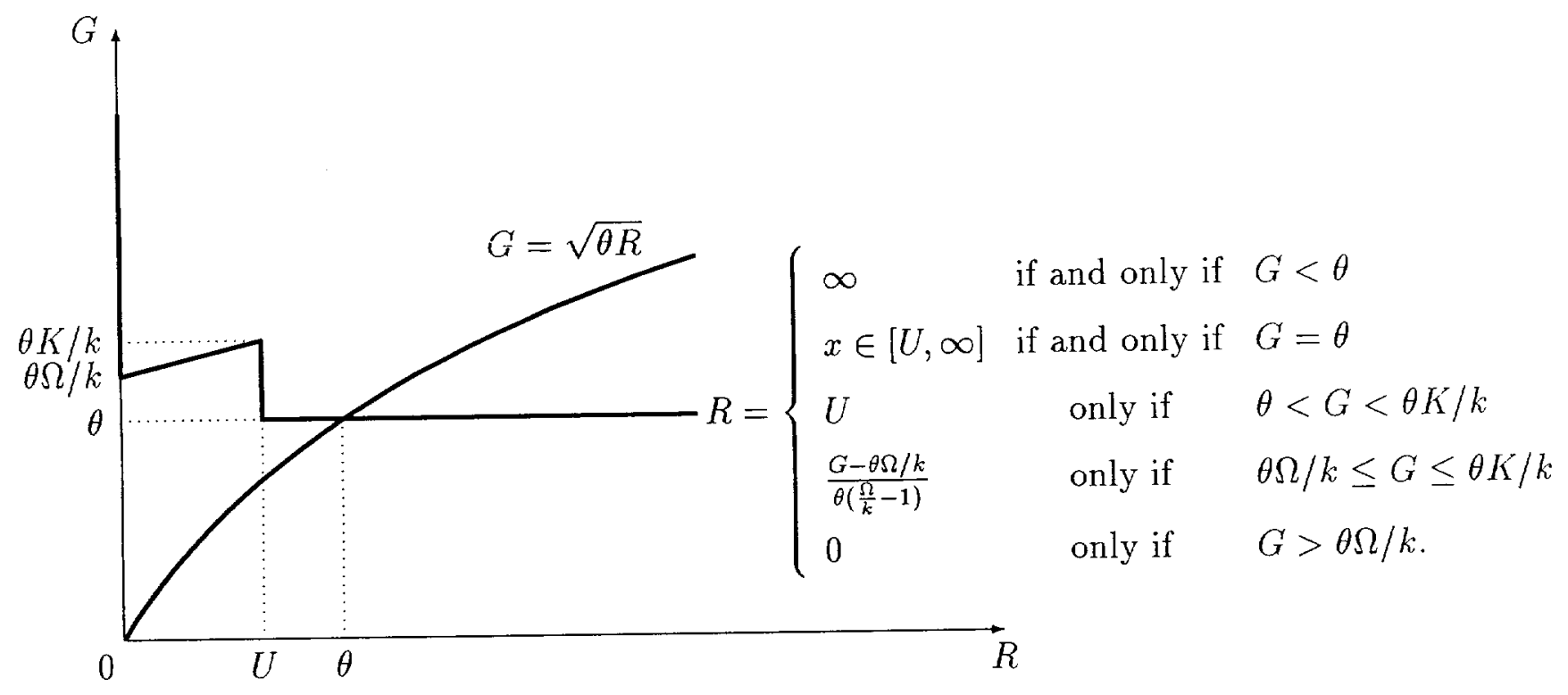

Figure 1: $R=\theta>U$

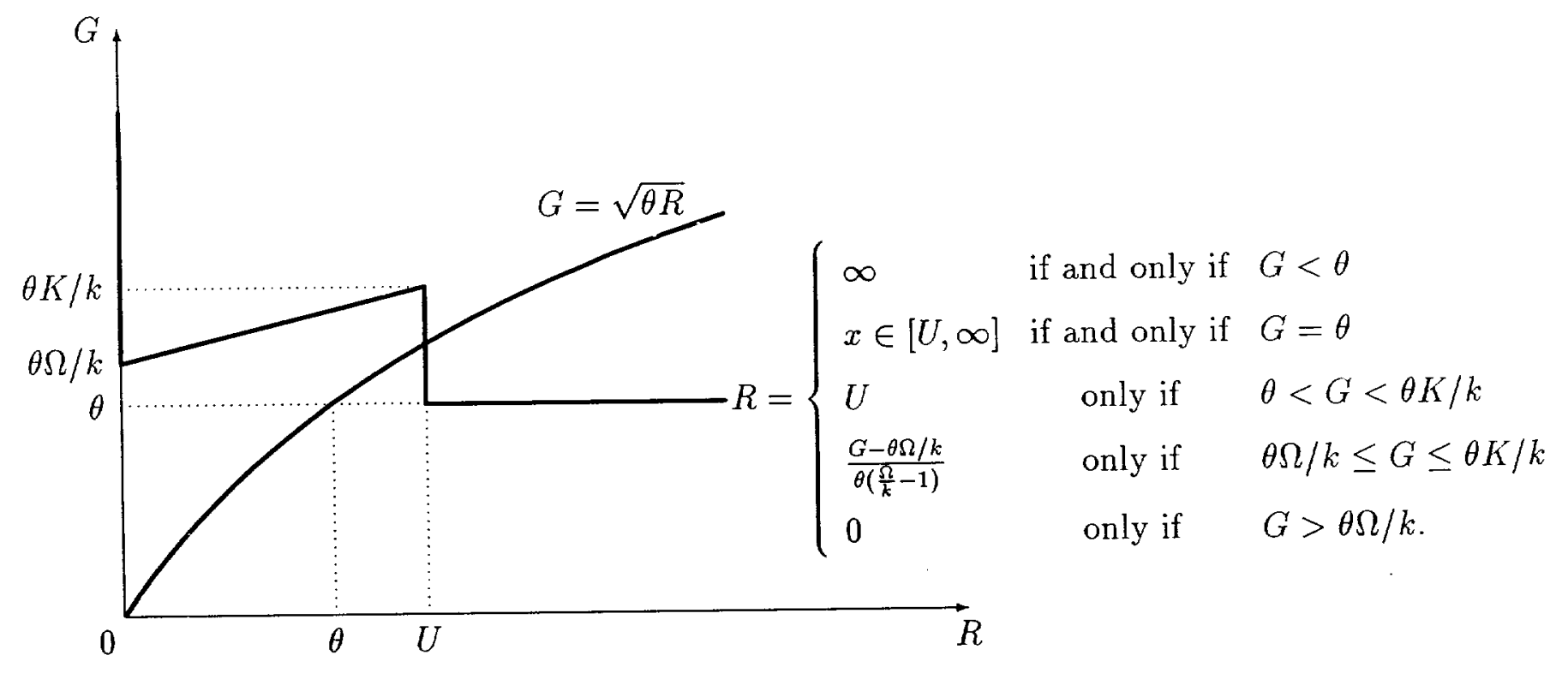

Figure 2: $R=U>\theta$ 
than $\theta$. In other words, regardless of the size of $U, R$ is not smaller than $\theta$. Furthermore, $R$ is equal to $\theta$ if and only if $U$ is not larger than $\theta .^{9}$

These two implications of equation (7) bring out the subtle nonmonotonic relation between $U$ and the equilibrium value of $R$. If the number of unprivileged people is so large that $U$ is larger than the parameter $\theta$, then in equilibrium $R$ is larger than $\theta$. But, if the number of unprivileged people were so small that $U$ were smaller than $\theta$, then in equilibrium $R$ would not be smaller than $\theta$. Rather, if $U$ were smaller than $\theta$, then in equilibrium enough of the privileged people would choose to be predators to make $R$ equal $\theta$.

\section{Rawlsian Distributions of Resources}

We focus the welfare analysis on deriving the interpersonal distribution of resources that is preferred according to the Rawlsian criterion. The Rawlsian criterion is to maximize the utility of the least advantaged person. In the present model, consumption is a cardinal index of utility.

Recall that the model assumes that the production technology is linear and that individual productive activities are independent, and that the model also abstracts from trade. Given this setup, if we were to ignore the possibility of predation, then average production and consumption would equal $\Omega$, independently of the interpersonal distribution of resources, and each person's consumption would equal that person's production. Conse-

${ }^{9}$ To see the logic of these implications, recall that, if $R$ were smaller than $\theta$, then according to equation (6) the chosen value of $G$ would be smaller than $\theta$. But, if $G$ were smaller than $\theta$, then $D$ would be larger than both $c$ and $C$, and, as indicated by equation (5), every person would prefer to be a predator. Thus, regardless of the size of $U, R$ smaller than $\theta$ would be a contradiction.

Also, recall that, if $R$ were equal to $\theta$, then according to equation (6) $G$ would be equal to $\theta$. But, if $G$ were equal to $\theta$, then $D$ would be larger than $c$, and, as indicated by equation (5), $R$ would be at least as large as $U$. Thus, if $U$ is larger than $\theta$, then $R$ cannot equal $\theta$. Conversely, given that $R$ cannot be smaller than $\theta$, if $U$ is larger than $\theta$, then $R$ is larger than $\theta$. 
quently, the fully egalitarian distribution of resources would imply equal production and consumption for every person and, hence, would maximize the production and consumption of the person who has the lowest consumption. Thus, abstracting from predation, the Rawlsian criterion would select the fully egalitarian distribution.

The fact that in equilibrium some people choose to be predators radically alters this conclusion. As suggested above, the key to understanding the welfare properties of the interpersonal distribution of resources in this model that allows predation is to observe that the choice by some people to be predators decreases aggregate consumption, both because the resources of predators are wasted by not being used productively and because any resources that producers allocate to guarding against predators reduces their production. In addition, predation increases the consumption of the unprivileged people relative to the consumption of the privileged people.

Equation (6) implies that the amount of resources allocated to guarding against predators is positively related to the number of predators. Thus, the negative effect of predation on aggregate consumption is an increasing function of both the number of people who choose to be predators and their endowment of resources, which is wasted.

Substituting for $p$ from equation (2) and for $G$ and $R$ from equations (6) and (7) into equations (3) and (4), we can calculate the consumption in equilibrium of privileged people and unprivileged people. Because privileged people in equilibrium either prefer to be producers or are indifferent between being producers and being predators, the consumption of every privileged person is given by

$$
C= \begin{cases}\frac{K}{(1+\theta)^{2}} & \text { for } \theta \geq U \\ \frac{K}{(1+\sqrt{\theta U})^{2}} & \text { for } U>\theta .\end{cases}
$$

Because unprivileged people in equilibrium either prefer to be predators or are indifferent between being predators or producers, the consumption of every unprivileged person is given 
by $^{10}$

$$
D= \begin{cases}\frac{K}{(1+\theta)^{2}} & \text { for } \theta \geq U \\ \frac{K \sqrt{\theta / U}}{(1+\sqrt{\theta U})^{2}} & \text { for } U>\theta .\end{cases}
$$

Equations (8) and (9) imply that, if $U$ is larger than $\theta$, then $D$ is smaller than $C$, but that, if $U$ is smaller than or equal to $\theta$, then $D$ is equal to $C$. Thus, for any

${ }^{10}$ If $R_{1}$ and $R_{2}$ exist, then equation (8) would become

$$
C= \begin{cases}\frac{K}{(1+\theta)^{2}} & \text { for } U \leq \theta \\ \frac{K}{(1+\sqrt{\theta U})^{2}} & \text { for } \theta<U \leq R_{1} \\ \frac{K}{\left(1+\sqrt{\theta R_{1}}\right)^{2}} & \text { for } R_{1}<U<R_{2} \\ \left\{\frac{K}{\left(1+\sqrt{\theta R_{1}}\right)^{2}}, \frac{K}{\left(1+\sqrt{\theta R_{2}}\right)^{2}}, \frac{K}{(1+\sqrt{\theta U})^{2}}\right\} & \text { for } U \geq R_{2},\end{cases}
$$

and equation (9) would become

$$
D= \begin{cases}\frac{K}{(1+\theta)^{2}} & \text { for } U \leq \theta \\ \frac{K \sqrt{\theta / U}}{(1+\sqrt{\theta U})^{2}} & \text { for } \theta<U \leq R_{1} \\ \frac{k}{\left(1+\sqrt{\theta R_{1}}\right)^{2}} & \text { for } R_{1}<U<R_{2} \\ \left\{\frac{k}{\left(1+\sqrt{\theta R_{1}}\right)^{2}}, \frac{k}{\left(1+\sqrt{\theta R_{2}}\right)^{2}}, \frac{K \sqrt{\theta / U}}{(1+\sqrt{\theta U})^{2}}\right\} & \text { for } U \geq R_{2} .\end{cases}
$$

Using these more general equations for $C$ and $D$, we can confirm that, because both $R_{1}$ and $R_{2}$ would be larger than $\theta$, assuming that $R_{1}$ and $R_{2}$ exist would not affect the results of the welfare analysis that follows. 
interpersonal distribution of resources the consumption of each unprivileged person, which is equal to $D$, is less than or equal to the consumption of each privileged person, which is equal to $C$. Accordingly, to apply the Rawlsian criterion in the present model we only have to determine what distribution of resources implies the maximum value of $D$.

From equation (1) we can relate $K$, the endowment of a privileged person, to $\Omega$, the average endowment of resources, and to $U$, the ratio of privileged people to unprivileged people, and to $k$, the endowment of an unprivileged person. Specifically,

$$
K=\Omega+U(\Omega-k) .
$$

Also, recall that in analysing the welfare properties of the interpersonal distribution of resources, we hold $\Omega$ fixed. Equation (10) implies that, given $\Omega$, the combination of $U$ and $k$ fully describes the interpersonal distribution of resources.

Substituting equation (10) into equation (9) we find that the combination $U=\theta$ and $k=$ 0 implies the maximum value of $D$. This maximum value of $D$ is $\frac{\Omega}{1+\theta}$. This result means that the consumption of the person who has the lowest consumption is maximized with a positive number of unprivileged people, each of whom has the minimum possible endowment of resources. With this unegalitarian distribution of resources the unprivileged people choose to be predators, the privileged people choose to be producers, and each unprivileged person appropriates from the privileged people an amount that is larger than what his or her consumption would be with any other interpersonal distribution of resources, including the fully egalitarian distribution. Equation (9) implies that with the fully egalitarian distribution of resources $D$ would be equal to $\frac{\Omega}{(1+\theta)^{2}}$. In addition, the unegalitarian Rawlsian distribution of resources results in equal consumption for the unprivileged and the privileged.

The result that the Rawlsian criterion selects the distribution of resources that has $U$ equal to $\theta$ and $k$ equal to zero is easy to explain. According to equation (7) the equilibrium ratio of predators to producers, $R$, is not smaller than $\theta$. Also, $R$ equals its minimum value of $\theta$ for all values of $U$ smaller than or equal to $\theta$. Thus, with $U$ equal to $\theta$ 
both the number of predators and the resulting amount of resources allocated to guarding against predators is minimized.

Furthermore, with $U$ equal to $\theta$ the unprivileged people prefer to be predators, whereas the privileged people are just indifferent between being producers and predators. Thus, with both $U$ and $R$ equal to $\theta$ all of the predators are unprivileged people, and each predator wastes only the endowment of resources of an unprivileged person. In addition, with $k$ equal to zero the amount of resources that each unprivileged predator wastes is also minimized. Accordingly, the combination $U=\theta$ and $k=0$ minimizes the negative effect of predation on the aggregate production of consumables and, hence, maximizes aggregate consumption.

Finally, because with $U$ equal to $\theta$ the privileged people are just indifferent between - being producers and predators, the consumption of privileged producers and unprivileged predators is equal. Thus, with $U$ equal to $\theta$ maximizing aggregate consumption maximizes consumption for every person and, hence, for the person with the lowest consumption.

\section{Pareto Efficient Distributions of Resources}

Because the Rawlsian distribution of resources $-U=\theta$ and $k=0-$ implies maximum consumption for the person with the lowest consumption, the Rawlsian distribution is also Pareto efficient. A Pareto efficient distribution of resources is a distribution such that no redistribution of resources would result in a Pareto improvement. A Pareto improvement is an increase in the net consumption of at least one person without a decrease in the net consumption of any other person.

In this model, if we were to ignore the possibility of predation, then all interpersonal distributions of resources would be Pareto efficient. Without the possibility of predation, any redistribution of resources would reduce somebody's consumption. Allowing for the possibility of predation, however, introduces the possibility that a redistribution of resources could result in a Pareto improvement.

As we have seen, with the fully egalitarian distribution of resources $D$ would be equal 
to $\frac{\Omega}{(1+\theta)^{2}}$, whereas with the Rawlsian distribution $D$ would be equal to $\frac{\Omega}{1+\theta}$. In addition, with either the fully egalitarian distribution or the Rawlsian distribution $C$ and $D$ would be equal. Thus, with the Rawlsian distribution of resources $C$ as well as $D$ would be higher than with the fully egalitarian distribution. In other words, not only does the fully egalitarian distribution of resources not satisfy the Rawlsian criterion of maximizing the consumption of the person with the lowest consumption, the fully egalitarian distribution of resources is not even Pareto efficient. In fact, the Rawlsian distribution of resources would result in a Pareto improvement over any distribution in which either $U$ is less than $\theta$ or $k$ is positive.

The Rawlsian distribution of resources is not the only Pareto efficient distribution. For example, equations (8) and (9) imply that with $U$ larger than $\theta$ the consumption of an unprivileged person, $D$, is smaller than the consumption of an privileged person, $C$. Thus, starting with $U$ larger than $\theta$, decreasing $U$ to $\theta$, by changing the status of some people from unprivileged to privileged would increase the consumption of these people. But, equation (8) also implies that for some distributions of resources in which $k$ is less than $\Omega$ and $U$ is larger than $\theta$ the consumption of a privileged person, $C$, is higher than $\frac{\Omega}{1+\theta}$, which is the level of $C$ associated with the Rawlsian distribution. Thus, starting with any of these distributions decreasing $U$ to $\theta$ would decrease the consumption of the remaining privileged people and, hence, would not result in a Pareto improvement. Accordingly, any of these distributions of resources is also a Pareto efficient distribution.

\section{Summary}

We have analysed a general-equilibrium model in which some people (the privileged) are well endowed with resources and other people (the unprivileged) are poorly endowed with resources and in which each person can choose to be either a producer or a predator. We began by determining how the equilibrium ratio of predators to producers depends on the technology of predation and on the interpersonal distribution of resources. Because a 
privileged person can produce more than an unprivileged person, predation is less attractive for the privileged than for the unprivileged. But, we found that the minimum equilibrium ratio of predators to producers depends only on the technology of production. If the ratio of unprivileged people to privileged were smaller than this minimum equilibrium ratio of predators to producers, then in equilibrium all of the unprivileged people as well as some of the privileged people would choose to be predators. We also found that the equilibrium ratio of predators to producers exceeds its minimum value if and only if the ratio of unprivileged people to privileged people is larger than this minimum value.

We then used these results to show how predation breaks the links between an economy's aggregate resource endowment and aggregate consumption and between the interpersonal distribution of endowments and the interpersonal distribution of consumption. Most interestingly, we found that with some people choosing to be predators the Rawlsian criterion of maximizing the consumption of the person with the lowest consumption selects an unegalitarian distribution of resources in which these people have only the minimum possible endowment of resources. In addition, the unegalitarian distribution of resources that satisfies the Rawlsian criterion results in equal consumption for unprivileged people and privileged people. These results obtain even though our model is such that, if we were to ignore the possibility of predation, then the Rawlsian criterion would select the fully egalitarian distribution of resources. But, allowing for predation, we found that the fully egalitarian distribution is not even Pareto efficient.

The key to understanding the welfare properties of the interpersonal distribution of resources in this model that allows predation was to observe that the negative effect of predation on aggregate consumption is an increasing function of both the number of people who choose to be predators and their endowment of resources, which is wasted. In addition, predation increases the consumption of the unprivileged people relative to the consumption of the privileged people.

With the unegalitarian distribution of resources that satisfies the Rawlsian criterion both 
the number of predators and the resulting amount of resources allocated to guarding against predators is minimized. Furthermore, all of the predators are unprivileged people, and each predator wastes only the endowment of resources of an unprivileged person. In addition, the amount of resources that each unprivileged predator wastes is also minimized. Accordingly, the unegalitarian distribution of resources that satisfies the Rawlsian criterion minimizes the negative effect of predation on the aggregate production of consumables and, hence, maximizes aggregate consumption. Finally, because with this distribution of resources the privileged people are just indifferent between being producers and predators, the consumption of privileged producers and unprivileged predators is equal. Thus, maximizing aggregate consumption maximizes consumption for every person and, hence, for the person with the lowest consumption. 


\section{References}

Brito, Dagobert and Intriligator, Michael. "Conflict, War, and Redistribution", American Political Science Review, 79, 1985, 943-957.

Grossman, Herschel I. and Kim, Minseong. "Swords or Plowshares? A Theory of the Security of Claims to Property," Journal of Political Economy, 103, December 1995, 1275-1288.

Grossman, Herschel I. and Kim, Minseong. "Predation and Production," in Michelle R. Garfinkel and Stergios Skaperdas, eds., The Political Economy of Conflict and Appropriation, New York: Cambridge University Press, 1996a.

Grossman, Herschel I. and Kim, Minseong. "Predation and Moral Decay," unpublished, April 1977.

Rawls, John. A Theory of Justice, Cambridge: Harvard University Press, 1971.

- Usher, Dan. "Theft as a Paradigm for Departures from Efficiency, "Oxford Economic Papers, 39, June 1987, 235-252; reprinted as Chapter III in Dan Usher, The Welfare Economics of Markets, Voting and Predation, Ann Arbor: The University of Michigan Press, 1992. 